PROCEEDINGS OF THE

AMERICAN MATHEMATICAL SOCIETY

Volume 138, Number 10, October 2010, Pages 3509-3520

S 0002-9939(10)10394-3

Article electronically published on May 10, 2010

\title{
MOTIVIC STRICT RING MODELS FOR $K$-THEORY
}

\author{
OLIVER RÖNDIGS, MARKUS SPITZWECK, AND PAUL ARNE ØSTVÆR
}

(Communicated by Brooke Shipley)

\begin{abstract}
It is shown that the $K$-theory of every noetherian base scheme of finite Krull dimension is represented by a commutative strict ring object in the setting of motivic stable homotopy theory. The adjective 'strict' is used here in order to distinguish between the type of ring structure we construct and one which is valid only up to homotopy. An analogous topological result follows by running the same type of arguments as in the motivic setting.
\end{abstract}

\section{INTRODUCTION}

Motivic homotopy theory can be viewed as an expansion of classical homotopy theory to an algebro-geometric setting. This has enabled the introduction of homotopy theoretic techniques in the study of generalized ring (co)homology theories for schemes, and as in classical algebra one studies these via modules and algebras. From this perspective, motives are simply modules over the motivic EilenbergMacLane ring spectrum [9, [10. The main purpose of this paper is to show that the $K$-theory of every noetherian base scheme of finite Krull dimension acquires strict ring object models in motivic homotopy theory, and thereby pave the way towards a classification of modules over $K$-theory. An example of a base scheme of particular interest is the integers. Working with some flabby smash product which only become associative, commutative and unital after passage to the motivic stable homotopy category is inadequate for our purposes. The paper is couched in terms of motivic symmetric spectra [4, following constructions introduced by Schwede [1] in the classical contexts of simplicial and topological symmetric spectra. Throughout the term ' $K$-theory' is short for homotopy algebraic $K$-theory.

Fix a noetherian base scheme $S$ of finite Krull dimension with multiplicative group scheme $\mathbf{G}_{\mathfrak{m}}$. Denote by $\mathrm{KGL}$ the ordinary motivic spectrum representing $K$-theory [14. As shown in [12 (see also 3 and [7), inverting a homotopy class $\beta \in \pi_{2,1} \Sigma^{\infty} \mathbf{B G}_{\mathfrak{m}+}$ in the motivic suspension spectrum of the classifying space of the multiplicative group scheme yields a natural isomorphism in the motivic stable homotopy category

$$
\Sigma^{\infty} \mathrm{BG}_{\mathfrak{m}+}\left[\beta^{-1}\right] \stackrel{\cong}{\longrightarrow} \mathrm{KGL} .
$$

We shall turn the Bott inverted model for $K$-theory into a commutative monoid $\mathrm{KGL}^{\beta}$ in the category of motivic symmetric spectra. To start with, the multiplicative structure of $\mathbf{G}_{\mathfrak{m}}$ induces a commutative monoid structure on the motivic

Received by the editors October 13, 2009 and, in revised form, January 19, 2010

2010 Mathematics Subject Classification. Primary 14F42, 55P43; Secondary 19E08.

(C)2010 American Mathematical Society

Reverts to public domain 28 years from publication 
symmetric suspension spectrum of the classifying space $\mathbf{B G}_{\mathfrak{m}+}$. A far more involved analysis dealing with an actual map rather than some homotopy class allows us to define $\mathrm{KGL}^{\beta}$ and eventually verify that it is a commutative monoid with the same homotopy type as $K$-theory. This part of the paper is self-contained. Several of the main techniques employed in the proof are of interest in their own right and can be traced back to constructions for symmetric spectra in [11.

When suitably adopted, the motivic argument also works in topological categories. The topological strict ring models appear to be new, even in the case of symmetric spectra [11].

The Bott element considered by Voevodsky in [14] is obtained from the virtual vector bundle

$$
\left[\mathcal{O}_{\mathbf{P}^{1}}\right]-\left[\mathcal{O}_{\mathbf{P}^{1}}(-1)\right]
$$

A key step in the construction of $\mathrm{KGL}^{\beta}$ is to interpret the same element, viewed in the pointed motivic unstable homotopy category, as an actual map between motivic spaces. In order to make this part precise we shall use a lax symmetric monoidal fibrant replacement functor for pointed motivic spaces. Fibrancy is a constant source for extra fun in abstract homotopy theory. The problem resolved in this paper is no exception in that respect. It is also worthwhile to emphasize the intriguing fact that $\beta$ does not play a role in the definition of the multiplicative structure of $\mathrm{KGL}^{\beta}$. However, the Bott element enters in the definition of the unit map $\mathbf{1} \rightarrow \mathrm{KGL}^{\beta}$, which is part of the monoid structure, and in the structure maps. In fact, up to some fibrant replacement, $\mathrm{KGL}^{\beta}$ is constructed fairly directly from $\Sigma^{\infty} \mathbf{B G}_{\mathfrak{m}+}$ by intertwining a map representing $\beta$ with the structure maps of the suspension spectrum of $\mathbf{B G}_{\mathfrak{m}+}$. On the level of homotopy groups this type of intertwining has the effect of inverting the Bott element. As a result, we deduce that $\mathrm{KGL}^{\beta}$ has the homotopy type of $K$-theory. The 'strictification method' can also be used to show that the periodized algebraic cobordism spectrum introduced in [3] acquires a strict ring model.

In 8$]$ it is shown that under a certain normalization assumption the ring structure on KGL in the motivic stable homotopy category is unique over the ring of integers $\mathbf{Z}$. For any base scheme $S$ the multiplicative structure pulls back to give a distinguished monoidal structure on KGL, which is unique according to [6, Remark 9.8 (ii)]. We show that the multiplicative structures on $\mathrm{KGL}^{\beta}$ and $\mathrm{KGL}$ coincide in the motivic stable homotopy category. The proof of this result is not formal. A key input is the subtle result that $K$-theory does not support any nontrivial phantom map in the motivic stable homotopy category over any base scheme. In turn, this is a consequence of the fundamental Landweber exactness theorem in motivic homotopy theory [6].

In [3] the setup of $\infty$-categories is used to note the existence of an $E_{\infty}$ or coherently homotopy commutative structure on $K$-theory. Work in progress suggests there exists a unique such structure. With the construction of $\mathrm{KGL}^{\beta}$ in hand one has a strict model for $K$-theory, and the strictness has the pleasing consequence that the model is amenable to a simpler homotopical study. Work on this subject has been initiated in [1. The existence of a suitable model structure for commutative motivic symmetric ring spectra suggests that the $E_{\infty}$-structure in [3] can be lifted to a strict commutative ring model for $K$-theory. 
Added in proof. A different construction of an associative motivic symmetric ring spectrum representing algebraic $K$-theory over a regular scheme has been obtained by Youngsoo Kim in his $\mathrm{PhD}$ thesis at UIUC.

\section{A STRICT MODEL}

The main focus of this section is the construction of a strict model for $K$-theory in the category of motivic symmetric spectra. Throughout we use the 'closed' motivic model structure in 8 with a view towards realization functors. An extensive background in motivic stable homotopy theory is not assumed.

The classifying space $\mathbf{B G}_{\mathfrak{m}}$ has terms $\mathbf{G}_{\mathfrak{m}}^{\times n}$ for $n \geq 0$ with the convention that its zeroth term is a point. Its face and degeneracy maps, which are defined in a standard way using diagonals and products, allow us to consider $\mathbf{B G}_{\mathfrak{m}}$ as a motivic space (that is, a simplicial presheaf on the Nisnevich site of the base scheme $S$ ).

Throughout we use the following standard notation: Let $S^{2,1}$ denote the motivic sphere defined as the smash product of the simplicial circle $S^{1,0}=\Delta^{1} / \partial \Delta^{1}$ with $\mathbf{G}_{\mathfrak{m}}$ pointed by its one-section. For $n \geq 2$ we set $S^{2 n, n}=S^{2 n-2, n-1} \wedge S^{2,1}$. When forming motivic spectra, we shall, for consistency with [11], smash with the suspension coordinate $S^{2,1}$ from the right.

In the Introduction it was recalled that the Bott element is a homotopy class

$$
\beta \in \pi_{2,1} \Sigma^{\infty} \mathbf{B G}_{\mathfrak{m}+} .
$$

As such, it is represented by a map of pointed motivic spaces

$$
S^{2 n+2, n+1} \rightarrow\left(\mathrm{BG}_{\mathfrak{m}+} \wedge S^{2 n, n}\right)^{\mathrm{fib}}
$$

for some $n$, where $(-)^{\text {fib }}$ denotes a fibrant replacement functor. The construction we give of $\mathrm{KGL}^{\beta}$ works for any such representative provided the fibrant replacement functor is lax symmetric monoidal. By Lemma 2.2 we may choose a fibrant replacement functor with the stated properties. As shown in the following, the situation at hand allows for an explicit construction of a map

$$
S^{4,2} \rightarrow\left(\mathrm{BG}_{\mathfrak{m}+} \wedge S^{2,1}\right)^{\mathrm{fib}}
$$

that represents the Bott element.

Before proceeding with the construction of the strict models we discuss fibrancy of the multiplicative group scheme and its classifying space, pertaining to the discussion of a fibrant replacement functor in the above.

Example 2.1. The classifying space of the multiplicative group scheme is sectionwise fibrant because it takes values in simplicial abelian groups. When $S$ is regular, then $\mathbf{G}_{\mathfrak{m}}$ is fibrant. However, as the following discussion shows, $\mathbf{B} \mathbf{G}_{\mathfrak{m}}$ is not fibrant.

The standard open covering of the projective line by affine lines yields an elementary distinguished square:

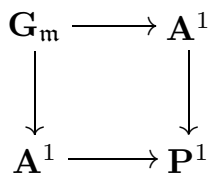

Let $P$ denote the homotopy pullback of the diagram

$$
\mathrm{BG}_{\mathfrak{m}}\left(\mathrm{A}^{1}\right) \longrightarrow \mathrm{BG}_{\mathfrak{m}}\left(\mathbf{G}_{\mathfrak{m}}\right) \longleftarrow \mathrm{BG}_{\mathfrak{m}}\left(\mathrm{A}^{1}\right)
$$


obtained by applying $\mathbf{B G}_{\mathfrak{m}}$ to (2.1). Then the homotopy fiber of the map $P \rightarrow$ $\mathbf{B G}_{\mathfrak{m}}\left(\mathbf{A}^{1}\right)$ is weakly equivalent to the homotopy fiber $F$ of $\mathbf{B G}_{\mathfrak{m}}\left(\mathbf{A}^{1}\right) \rightarrow \mathbf{B G}_{\mathfrak{m}}\left(\mathbf{G}_{\mathfrak{m}}\right)$. For a ring $R$, let $R^{\times}$denote its multiplicative group of units. With these definitions there exist induced exact sequences of homotopy groups

$$
0 \longrightarrow \pi_{1} P \longrightarrow \mathcal{O}_{S}^{\times}=\mathcal{O}_{S}\left[t^{-1}\right]^{\times} \longrightarrow \pi_{0} F \longrightarrow \pi_{0} P \longrightarrow 0
$$

and

$$
0 \longrightarrow \mathcal{O}_{S}^{\times}=\mathcal{O}_{S}[t]^{\times} \longrightarrow \mathcal{O}_{S}\left[t, t^{-1}\right]^{\times} \longrightarrow \pi_{0} F \longrightarrow 0 .
$$

(Here $t$ is an indeterminate.) Hence $\mathcal{O}_{S}\left[t^{-1}\right]^{\times} \rightarrow \pi_{0} F$ cannot be surjective. Thus $P$ is not connected and, in particular, not weakly equivalent to $\mathbf{B G}_{\mathfrak{m}}\left(\mathbf{P}^{1}\right)=\mathbf{B O}_{S}^{\times}$. This shows that $\mathbf{B G}_{\mathfrak{m}}$ does not satisfy the Nisnevich fibrancy condition; see [2], 4, [5].

We write

$$
i: S^{1,0} \wedge \mathbf{G}_{\mathfrak{m}} \rightarrow \mathbf{B G}_{\mathfrak{m}}
$$

for the inclusion of the 1-skeleton $\mathbf{G}_{\mathfrak{m}}$ into the classifying space $\mathbf{B G}_{\mathfrak{m}}$. Let

$$
c: S^{1,0} \wedge \mathbf{G}_{\mathfrak{m}} \rightarrow \mathrm{BG}_{\mathfrak{m}}
$$

denote the constant map. Via the motivic weak equivalences $S^{1,0} \wedge \mathbf{G}_{\mathfrak{m}} \simeq \mathbf{P}^{1}$ and $\mathbf{B G}_{\mathfrak{m}} \simeq \mathbf{P}^{\infty}$, the map $i$ can be identified in the pointed motivic unstable homotopy category with the inclusion

$$
\mathbf{P}^{1} \rightarrow \mathbf{P}^{\infty}
$$

In homogeneous coordinates the inclusion map is given by

$$
[x: y] \mapsto[x: y: 0: \cdots] .
$$

Similarly, the map $c$ coincides with the canonical composite map

$$
\mathbf{P}^{1} \rightarrow S \rightarrow \mathbf{P}^{\infty}
$$

given by

$$
[x: y] \mapsto[1: 0: \cdots] .
$$

Adding a disjoint base point to the classifying space of $\mathbf{G}_{\mathfrak{m}}$ yields pointed maps

$$
i_{+}, c_{+}: S^{2,1} \rightarrow \mathrm{BG}_{\mathfrak{m}+}
$$

for the base point of $\mathbf{B G}_{\mathfrak{m}}$. Now in order to move the base point around in $\mathbf{B G}_{\mathfrak{m}+}$ we take the unreduced suspension of both these maps. Recall that the unreduced suspension of a motivic space $A$ is defined as the pushout

$$
S(A)=A \times \Delta^{1} \cup_{A \times \partial \Delta^{1}} \partial \Delta^{1} .
$$

One can view it as a pointed motivic space by the image of $0 \in \partial \Delta^{1}$. With this definition, the unreduced suspensions of the maps $i_{+}$and $c_{+}$are pointed with respect to the image of $(+, 0)$ in their targets. If $A$ is pointed, the canonical map $q: S(A) \rightarrow \Sigma A$ to the reduced suspension is a weak equivalence. Hence there exists a map of pointed motivic spaces

$$
S^{4,2} \rightarrow\left(S\left(S^{2,1}\right) \wedge \mathbf{G}_{\mathfrak{m}}\right)^{\text {fib }}
$$

lifting the inverse of the map $q \wedge \mathbf{G}_{\mathfrak{m}}$ in the pointed motivic unstable homotopy category. By composing we end up with the two pointed maps

$$
i^{\beta}, c^{\beta}: S^{4,2} \stackrel{\sim}{\longrightarrow}\left(S\left(S^{2,1}\right) \wedge \mathbf{G}_{\mathfrak{m}}\right)^{\mathrm{fib}} \rightrightarrows\left(S\left(\mathbf{B G}_{\mathfrak{m}+}\right) \wedge \mathbf{G}_{\mathfrak{m}}\right)^{\mathrm{fib}} \stackrel{\sim}{\longrightarrow}\left(\mathbf{B G}_{\mathfrak{m}+} \wedge S^{2,1}\right)^{\mathrm{fib}} .
$$


As a first approximation of the Bott element $\beta$ we consider the analog of the virtual vector bundle $\left[\mathcal{O}_{\mathbf{P}^{1}}\right]-\left[\mathcal{O}_{\mathbf{P}^{1}}(-1)\right]$ in the pointed motivic unstable homotopy category

$$
c^{\beta}-i^{\beta}: S^{4,2} \rightarrow\left(\mathbf{B G}_{\mathfrak{m}} \wedge S^{2,1}\right)^{\mathrm{fib}} .
$$

In order to form the difference map we use the fact that $S^{4,2}$ is a (two-fold) simplicial suspension and therefore a cogroup object in the pointed motivic unstable homotopy category. Note, however, that $c^{\beta}$ represents the trivial map because it factors through the base point. By appealing to the motivic model structure it follows that (2.2) lifts to a 'strict' motivic Bott map

$$
S^{4,2} \rightarrow\left(\mathrm{BG}_{\mathfrak{m}}+\wedge S^{2,1}\right)^{\mathrm{fib}}
$$

between pointed motivic spaces. Here we use the fact that the motivic sphere is cofibrant in the closed motivic model structure.

The fibrancy caveat above requires us to replace the suspension object $\Sigma^{\infty} \mathbf{B G}_{\mathfrak{m}+}$ with a levelwise fibrant motivic spectrum. An arbitrary such replacement need not preserve commutative monoids. The following lemma addresses this issue.

Lemma 2.2. There exists a lax symmetric monoidal fibrant replacement functor Id $\rightarrow F$ on the category of pointed motivic spaces.

Proof. The straightforward simplicial presheaf analog of [5, Theorem 2.1.66] provides a lax symmetric monoidal fibrant replacement functor $\mathrm{Ex}^{\infty}$ for the local model structure on any site of finite type. Moreover, the singular endofunctor Sing ${ }_{*}$ [5] constructed by means of the standard cosimplicial $S$-scheme $\Delta_{\mathbf{A}^{1}}^{\bullet}$ with terms

$$
\Delta_{\mathbf{A}^{1}}^{n}=S \times_{\operatorname{Spec}(\mathbf{Z})} \operatorname{Spec}\left(\mathbf{Z}\left[x_{0}, \ldots, x_{n}\right] / x_{0}+\cdots+x_{i}-1\right)
$$

is strict symmetric monoidal, because it commutes with limits and colimits. Thus the lemma follows by using the iterated construction

$$
\mathrm{Ex}^{\infty} \circ\left(\mathrm{Ex}^{\infty} \circ \mathrm{Sing}_{*}\right)^{\omega} \circ \mathrm{Ex}^{\infty}
$$

as the fibrant replacement functor [5, Lemma 3.2.6]. In this definition, $\omega$ denotes the cardinality of the natural numbers.

Corollary 2.3. The motivic symmetric spectrum $F\left(\Sigma^{\infty} \mathbf{B G}_{\mathfrak{m}+}\right)$ obtained by applying the functor $F$ levelwise to $\Sigma^{\infty} \mathbf{B G}_{\mathfrak{m}+}$ is a commutative monoid.

Proof. The assertion follows immediately by combining Lemma 2.2 and the fact that $\mathbf{G}_{\mathfrak{m}}$ is a commutative group scheme.

Corollary 2.4. There exists a motivic Bott map between pointed motivic spaces

$$
b: S^{4,2} \rightarrow F\left(\mathrm{BG}_{\mathfrak{m}}+\wedge S^{2,1}\right)
$$

that represents the difference map

$$
c^{\beta}-i^{\beta}
$$

in the pointed motivic unstable homotopy category. The map $b$ is central in the sense that the diagram

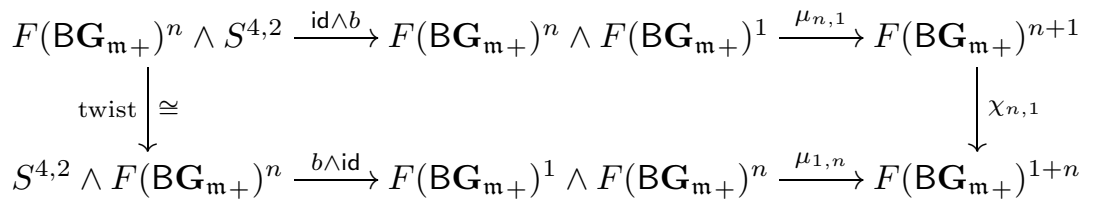


commutes. Here $F\left(\mathbf{B G}_{\mathfrak{m}_{+}}\right)^{k}$ is short for $F\left(\mathbf{B G}_{\mathfrak{m}_{+}} \wedge S^{2 k, k}\right)$ and $\chi_{n, 1}$ denotes the cyclic permutation $(1,2, \ldots, n, n+1)$.

Proof. This is immediate from Corollary 2.3 and the commutativity of $\mathbf{G}_{\mathfrak{m}}$.

With these preliminary results in hand we are ready to construct a strict ring model for $K$-theory. In the following we shall adopt constructions for symmetric spectra given in Schwede's manuscript [1] to the setting of motivic symmetric spectra. Let $\Omega^{2 n, n}$ denote the right adjoint of the suspension functor $-\wedge S^{2 n, n}$ on pointed motivic spaces.

Define $\mathrm{KGL}^{\beta}$ to be the motivic symmetric spectrum with constituent spaces

$$
\mathrm{KGL}_{n}^{\beta}=\Omega^{4 n, 2 n} F\left(\mathrm{BG}_{\mathfrak{m}+} \wedge S^{4 n, 2 n}\right) .
$$

The group $\Sigma_{n}$ acts on $S^{4 n, 2 n}$ and therefore also on $F\left(\mathrm{BG}_{\mathfrak{m}+} \wedge S^{4 n, 2 n}\right)$ via restriction along the diagonal embedding

$$
\Delta_{n}: \Sigma_{n} \rightarrow \Sigma_{2 n}
$$

defined for $1 \leq j \leq 2$ and $1 \leq i \leq n$ by setting

$$
\Delta_{n}(\sigma)(j+2(i-1))=j+2(\sigma(i)-1) .
$$

Now the $\Sigma_{n}$-action on the $(4 n, 2 n)$-loop space $\mathrm{KGL}_{n}^{\beta}$ is defined by conjugation. That is, for elements $\sigma \in \Sigma_{n}$ and $\phi \in \mathrm{KGL}_{n}^{\beta}$ define

$$
\sigma \cdot \phi(-)=\sigma\left(\phi\left(\sigma^{-1}(-)\right)\right)
$$

Let

$$
\mu_{m, n}: F\left(\mathbf{B G}_{\mathfrak{m}_{+}} \wedge S^{2 m, m}\right) \wedge F\left(\mathbf{B G}_{\mathfrak{m}_{+}} \wedge S^{2 n, n}\right) \rightarrow F\left(\mathbf{B G}_{\mathfrak{m}+} \wedge S^{2(m+n), m+n}\right)
$$

denote the maps comprising the multiplicative part of the monoid structure on

$$
F\left(\Sigma^{\infty} \mathbf{B G}_{\mathfrak{m}_{+}}\right) .
$$

Define the multiplication map

$$
\mathrm{KGL}_{m}^{\beta} \wedge \mathrm{KGL}_{n}^{\beta} \rightarrow \mathrm{KGL}_{m+n}^{\beta}
$$

by

$$
f \wedge g \mapsto \mu_{2 m, 2 n} \circ(f \wedge g) .
$$

The multiplication map (2.3) is strictly associative due to the strict associativity of the smash product and the multiplicative structure on $F\left(\Sigma^{\infty} \mathbf{B G}_{\mathfrak{m}_{+}}\right)$.

Moreover, (2.3) is $\Sigma_{m} \times \Sigma_{n}$-equivariant due to the equivariance of the multiplicative structure on $F\left(\Sigma^{\infty} \mathbf{B G}_{\mathfrak{m}_{+}}\right)$and the compatibility relation

$$
\Delta_{m}(\sigma) \times \Delta_{n}\left(\sigma^{\prime}\right)=\Delta_{m+n}\left(\sigma \times \sigma^{\prime}\right)
$$

for the diagonal embeddings $\Delta_{k}: \Sigma_{k} \rightarrow \Sigma_{2 k}$ (in our cases of interest $k=m, n, m+$ $n)$.

Let

$$
F\left(\mathrm{BG}_{\mathfrak{m}+} \wedge S^{2 n, n}\right) \rightarrow \mathrm{KGL}_{n}^{\beta}=\Omega^{4 n, 2 n} F\left(\mathrm{BG}_{\mathfrak{m}+} \wedge S^{4 n, 2 n}\right)
$$

be the adjoint of the composite map of

$$
\begin{aligned}
F\left(\mathrm{BG}_{\mathfrak{m}+} \wedge S^{2 n, n}\right) \wedge S^{4 n, 2 n} \stackrel{\text { id } \wedge b^{\wedge n}}{\longrightarrow} F\left(\mathrm{BG}_{\mathfrak{m}+} \wedge S^{2 n, n}\right) \wedge & F\left(\mathrm{BG}_{\mathfrak{m}+} \wedge S^{2 n, n}\right) \\
\downarrow \mu_{n, n} & \downarrow \\
F\left(\mathrm{BG}_{\mathfrak{m}+}\right. & \left.\wedge S^{4 n, 2 n}\right)
\end{aligned}
$$


and the map

$$
F\left(\mathrm{BG}_{\mathfrak{m}+} \wedge S^{4 n, 2 n}\right) \rightarrow F\left(\mathrm{BG}_{\mathfrak{m}+} \wedge S^{4 n, 2 n}\right)
$$

given by the permutation $\sigma \in \Sigma_{2 n}$ defined by

$$
\sigma(i)= \begin{cases}1+2(i-1), & 1 \leq i \leq n, \\ 2+2(k-1), & i=n+k, 1 \leq k \leq n .\end{cases}
$$

These maps assemble into a map between motivic symmetric ring spectra,

$$
F\left(\Sigma^{\infty} \mathrm{BG}_{\mathfrak{m}+}\right) \rightarrow \mathrm{KGL}^{\beta}
$$

In fact the structure maps

$$
\mathrm{KGL}_{n}^{\beta} \wedge S^{2,1} \rightarrow \mathrm{KGL}_{n+1}^{\beta}
$$

and the unit map of $\mathrm{KGL}^{\beta}$ are obtained from the above and the unit map of $F\left(\Sigma^{\infty} \mathbf{B G}_{\mathfrak{m}+}\right)$.

Lemma 2.5. The motivic symmetric spectrum $\mathrm{KGL}^{\beta}$ is a commutative monoid.

Proof. The commutativity constraint

$$
\mu_{2 m, 2 n} \circ(f \wedge g)=\mu_{2 n, 2 m} \circ(g \wedge f)
$$

follows because $\mathbf{G}_{\mathfrak{m}}$ is a commutative group scheme.

\section{The homotopy TYPE OF $\mathrm{KGL}^{\beta}$}

In this section we finish the proof of our main result and elaborate further on some closely related results. First we prepare for the comparison of $\mathrm{KGL}^{\beta}$ with the homotopy colimit of the Bott tower introduced in [12.

Let $\operatorname{sh}(-)$ denote the shifted motivic symmetric spectrum functor defined by

$$
\operatorname{sh}(\mathrm{E})_{n}=\mathrm{E}_{1+n}
$$

Its structure maps are induced from the ones for $E$ by reindexing. The $\Sigma_{n}$-action on the $n$th term of $\operatorname{sh}(\mathrm{E})$ is determined by the injection $(1 \times-): \Sigma_{n} \rightarrow \Sigma_{1+n}$ given by

$$
(1 \times \sigma)(i)= \begin{cases}1, & i=1 \\ \sigma(i-1)+1, & i \neq 1 .\end{cases}
$$

For our purposes the main application of the shift functor is to introduce the notion of a semistable motivic symmetric spectrum.

There exists a natural map

$$
\phi(\mathrm{E}): S^{2,1} \wedge \mathrm{E} \rightarrow \operatorname{sh}(\mathrm{E}) .
$$

In level $n$ it is defined as the composite map

$$
S^{2,1} \wedge \mathrm{E}_{n} \cong \mathrm{E}_{n} \wedge S^{2,1} \rightarrow \mathrm{E}_{n+1} \rightarrow \mathrm{E}_{1+n}
$$

of the twist isomorphism, the $n$th structure map of $\mathrm{E}$ and the cyclic permutation

$$
\chi_{n, 1}=(1,2, \ldots, n, n+1) .
$$

Using only the structure maps of $E$ would not give a map of motivic symmetric spectra. The map (3.1) is not a stable weak equivalence in general.

Definition 3.1. A motivic symmetric spectrum $E$ is called semistable if (3.1) is a stable weak equivalence of underlying (nonsymmetric) motivic spectra. 
Proposition 3.2. Let $\mathrm{E}$ be a motivic symmetric spectrum such that for every $n$ and every permutation $\sigma \in \Sigma_{n}$ with sign $\operatorname{sgn}(\sigma)=1$ the action of $\sigma$ on $\mathrm{E}_{n}$ coincides with the identity in the pointed motivic unstable homotopy category. Then $\mathrm{E}$ is semistable.

Proof. We may assume that E is levelwise fibrant. Then the standard natural stabilization construction $Q$ gives a stably fibrant replacement of E. Recall that in level $n$,

$$
Q(\mathrm{E})_{n}=\operatorname{colim}_{k}\left(\mathrm{E}_{n} \rightarrow \Omega^{2,1} \mathrm{E}_{n+1} \rightarrow \cdots \rightarrow \Omega^{2 k, k} \mathrm{E}_{n+k} \rightarrow \cdots\right),
$$

where the colimit is taken over the structure maps. It suffices to show that $Q(\phi(\mathrm{E}))$ is a levelwise weak equivalence. The assumption on $\mathrm{E}$ implies the composite map

$$
\begin{gathered}
\Omega^{2 k, k} F\left(S^{2,1} \wedge \mathrm{E}_{n+k}\right) \stackrel{\Omega^{2 k, k} F\left(\phi(\mathrm{E})_{n}\right)}{\longrightarrow} \Omega^{2 k, k} F\left(\operatorname{sh}(\mathrm{E})_{n+k}\right) \\
\downarrow \text { can } \\
\Omega^{2 k+4, k+2} F\left(S^{2,1} \wedge \operatorname{sh}(\mathrm{E})_{n+k+1}\right)
\end{gathered}
$$

coincides with the canonical map

$$
\Omega^{2 k, k} F\left(S^{2,1} \wedge \mathrm{E}_{n+k}\right) \stackrel{\text { can }}{\longrightarrow} \Omega^{2 k+4, k+2} F\left(S^{2,1} \wedge \mathrm{E}_{n+k+2}\right)
$$

in the pointed motivic unstable homotopy category. The canonical maps denoted by 'can' appear implicitly in (3.2). Thus $\phi(E)$ induces a weak equivalence on colimits

$$
Q\left(F\left(S^{2,1} \wedge \mathrm{E}\right)\right)_{n} \rightarrow Q(F(\operatorname{sh}(\mathrm{E})))_{n}
$$

for every $n$.

Example 3.3. The motivic symmetric spectrum $\Sigma^{\infty} \mathbf{B G}_{\mathfrak{m}+}$ is semistable. This follows from Proposition 3.2 because the even permutations are homotopic to the identity map on the motivic spheres. For the same reason, the motivic symmetric spectrum $\mathrm{KGL}^{\beta}$ is semistable.

Theorem 3.4. Let $\mathrm{E}$ be a semistable motivic symmetric spectrum, and let $U$ denote the right Quillen functor to motivic spectra that forgets the symmetric group actions. Then the value of the total right derived functor of $U$ at $\mathrm{E}$ is $U(\mathrm{E})$.

Proof. We may assume that $\mathrm{E}$ is cofibrant and levelwise fibrant. Let $R^{\infty} \mathrm{E}$ denote the colimit of the sequence

$$
\mathrm{E} \stackrel{\phi(\mathrm{E})^{\star}}{\longrightarrow} \Omega^{2,1}(\operatorname{sh}(\mathrm{E})) \stackrel{\Omega^{2,1}\left(\operatorname{sh}\left(\phi(\mathrm{E})^{\star}\right)\right)}{\longrightarrow} \cdots
$$

in the category of motivic symmetric spectra. Here $\phi(E)^{\star}$ is the adjoint of the map $\phi(\mathrm{E})$ defined in (3.1). By assumption $U(\phi(\mathrm{E}))$ is a stable weak equivalence of motivic spectra. Since $U$ commutes with the functors $S^{2,1} \wedge-$ and $\Omega^{2,1}$ forming a Quillen equivalence on motivic spectra, the derived adjoint

$$
U(\mathrm{E}) \rightarrow \Omega^{2,1}(U(\operatorname{sh}(\mathrm{E}))) \rightarrow \Omega^{2,1}\left((U \operatorname{sh}(\mathrm{E}))^{\mathrm{fib}}\right)
$$

is a stable weak equivalence. The stably fibrant replacement functor $Q$ commutes with $\Omega^{2,1}$ due to the finiteness of $S^{2,1}$. Thus the map

$$
\Omega^{2,1}(U(\operatorname{sh}(\mathrm{E}))) \rightarrow \Omega^{2,1} Q(U(\operatorname{sh}(\mathrm{E})))
$$

is a stable weak equivalence. It follows that $U\left(\phi(\mathrm{E})^{\star}\right)$ is also a stable weak equivalence of motivic spectra. 
Hence the canonical map $\mathrm{E} \rightarrow R^{\infty} \mathrm{E}$ is a stable weak equivalence of underlying motivic spectra. By [10, Theorem 18] the same map is also a stable weak equivalence of motivic symmetric spectra. Since $R^{\infty} \mathrm{E}$ is stably fibrant in the category of motivic symmetric spectra by construction, it gives a fibrant replacement of $\mathrm{E}$. The result follows from this since

$$
U(\mathrm{E}) \rightarrow U\left(R^{\infty} \mathrm{E}\right)
$$

is a stable weak equivalence.

Define

$$
a: F\left(\Sigma^{\infty} \mathbf{B G}_{\mathfrak{m}+}\right) \rightarrow \Omega^{4,2} \operatorname{sh}\left(F\left(\Sigma^{\infty} \mathbf{B G}_{\mathfrak{m}+}\right)\right)
$$

to be the adjoint of the map

$$
S^{4,2} \wedge F\left(\Sigma^{\infty} \mathrm{B}_{\mathbf{G}_{\mathfrak{m}}}\right) \rightarrow \operatorname{sh}\left(F\left(\Sigma^{\infty} \mathrm{B}_{\mathbf{G}_{\mathfrak{m}}}\right)\right) .
$$

In level $n$ the latter is the composite map

$S^{4,2} \wedge F\left(\Sigma^{\infty} \mathbf{B G}_{\mathfrak{m}_{+}}\right)_{n} \stackrel{b \wedge \text { id }}{\longrightarrow} F\left(\Sigma^{\infty} \mathbf{B G}_{\mathfrak{m}_{+}}\right)_{1} \wedge F\left(\Sigma^{\infty} \mathbf{B}_{\mathbf{G}_{\mathfrak{m}}}\right)_{n} \stackrel{\mu_{1, n}}{\longrightarrow} F\left(\Sigma^{\infty} \mathbf{B G}_{\mathfrak{m}+}\right)_{1+n}$.

Corollary 3.5. In the motivic stable homotopy category there exists an isomorphism between the homotopy colimit of the diagram of motivic symmetric spectra

$$
F\left(\Sigma^{\infty} \mathbf{B G}_{\mathfrak{m}_{+}}\right) \stackrel{a}{\rightarrow} \Omega^{4,2} \operatorname{sh}\left(F\left(\Sigma^{\infty} \mathbf{B G}_{\mathfrak{m}+}\right)\right) \stackrel{\Omega^{4,2} \operatorname{sh}(a)}{\longrightarrow} \cdots
$$

and the homotopy colimit $\Sigma^{\infty} \mathbf{B G}_{\mathfrak{m}+}\left[\beta^{-1}\right]$ of the Bott tower

$$
\Sigma^{\infty} \mathbf{B G}_{\mathfrak{m}+} \stackrel{\beta}{\rightarrow} \Sigma^{-2,-1} \Sigma^{\infty} \mathbf{B G}_{\mathfrak{m}+} \stackrel{\Sigma^{-2,-1} \beta}{\rightarrow} \cdots .
$$

Proof. Due to semistability of $F\left(\Sigma^{\infty} \mathbf{B G}_{\mathfrak{m}_{+}}\right)$, established in Example 3.3, we may identify $\Omega^{4,2} \operatorname{sh}\left(F\left(\Sigma^{\infty} \mathbf{B G}_{\mathfrak{m}_{+}}\right)\right)$with $\Omega^{4,2} F\left(S^{2,1} \wedge F\left(\Sigma^{\infty} \mathbf{B G}_{\mathfrak{m}_{+}}\right)\right)$and thus with $\Omega^{2,1} F\left(\Sigma^{\infty} \mathbf{B G}_{\mathfrak{m}+}\right)$ up to stable weak equivalence. The result follows since $a$ lifts the multiplication by the Bott element map (by construction).

Theorem 3.6. The motivic symmetric spectrum $\mathrm{KGL}^{\beta}$ has the homotopy type of the Bott inverted motivic spectrum $\Sigma^{\infty} \mathbf{B G}_{\mathfrak{m}+}\left[\beta^{-1}\right]$.

Proof. Corollary 3.5 identifies the Bott inverted motivic spectrum $\Sigma^{\infty} \mathbf{B G}_{\mathfrak{m}+}\left[\beta^{-1}\right]$ with the homotopy colimit of the diagram

$$
F\left(\Sigma^{\infty} \mathrm{BG}_{\mathfrak{m}+}\right) \stackrel{a}{\rightarrow} \Omega^{4,2} \operatorname{sh}\left(F\left(\Sigma^{\infty} \mathrm{BG}_{\mathfrak{m}+}\right)\right) \stackrel{\Omega^{4,2} \operatorname{sh}(a)}{\longrightarrow} \cdots
$$

Since the loop and shift functors appearing in (3.4) preserve semistability, it follows that the terms are semistable. Next we shall identify the homotopy colimit of (3.4) with $\mathrm{KGL}^{\beta}$. In effect, note that leaving the symmetric groups actions aside, $\mathrm{KGL}^{\beta}$ is the diagonal of the diagram of motivic symmetric spectra in (3.4). Example 3.3 and Theorem 3.4 show that the value of the right derived functor of $U$ at $\mathrm{KGL}^{\beta}$ is given by forgetting the group actions on $\mathrm{KGL}^{\beta}$. Hence there exists an abstract isomorphism between $\mathrm{KGL}^{\beta}$ and $\Sigma^{\infty} \mathbf{B G}_{\mathfrak{m}+}\left[\beta^{-1}\right]$ in the motivic stable homotopy category.

Remark 3.7. By [3], the periodized algebraic cobordism spectrum PMGL admits a model obtained by inflicting Bott periodicity in the suspension spectrum of $B \mathbf{G L}_{+}$. There exists a strict commutative model for the suspension spectrum of BGL +3 , Proposition 5.4], which is semistable according to Proposition [3.2. Semistability is needed and appears to be implicit in the proof of [3, Proposition 5.4]. Thus the constructions in this paper also produce a strict ring model $\mathrm{PMGL}^{\beta}$ for the 
periodized algebraic cobordism spectrum. Moreover, the determinant map GL $\rightarrow$ $\mathbf{G}_{\mathfrak{m}}$ induces a strict ring map $\mathrm{PMGL}^{\beta} \rightarrow \mathrm{KGL}^{\beta}$.

Remark 3.8. Applying the arguments in this paper to Kan's (lax symmetric monoidal) fibrant replacement functor for simplicial sets and the Bott element in $\pi_{2} \Sigma^{\infty} \mathrm{BC}^{\times}$yields a commutative symmetric ring spectrum with the homotopy type of topological unitary $K$-theory. More generally, for $A$ an abelian compact Lie group, the same argument applies to the Bott inverted model for $A$-equivariant unitary topological $K$-theory in 13 . We leave further details to the interested reader.

Next we discuss in broad strokes a motivic functor model for $K$-theory. There exists a strict symmetric monoidal functor from motivic symmetric spectra to motivic functors

$$
i^{*}: \text { MSS } \rightarrow \text { MF }
$$

for the base scheme $S$, which is part of the Quillen equivalence in [2, Theorem 3.32]. Therefore, the image $i^{*} \mathrm{KGL}^{\beta}$ of the motivic symmetric spectrum model for $K$ theory yields a commutative monoid in the category of motivic functors. However, since the motivic symmetric spectrum $\mathrm{KGL}^{\beta}$ need not be cofibrant, it is unclear whether $i^{*} \mathrm{KGL}^{\beta}$ represents homotopy algebraic $K$-theory in the homotopy category of motivic functors. A cofibrant replacement

$$
C \mathrm{KGL}^{\beta} \stackrel{\sim}{\longrightarrow} \mathrm{KGL}^{\beta}
$$

in the category of motivic symmetric ring spectra produces a (possibly noncommutative) motivic symmetric ring spectrum $C \mathrm{KGL}^{\beta}$ such that the associated motivic ring functor $i^{*} C \mathrm{KGL}^{\beta} \in \mathrm{MF}$ represents homotopy algebraic $K$-theory in the homotopy category of motivic functors.

\section{Multiplicative structure}

Theorem 4.1. The multiplicative structures on $\mathrm{KGL}^{\beta}$ and $\mathrm{KGL}$ coincide in the motivic stable homotopy category.

Proof. The proof proceeds by showing there is a commutative diagram of monoids

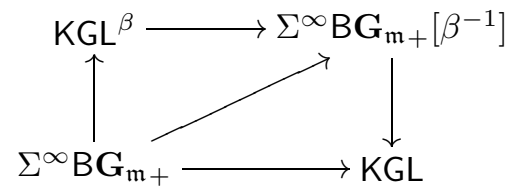

in the motivic stable homotopy category.

On the level of bigraded homology theories there is a commutative diagram:

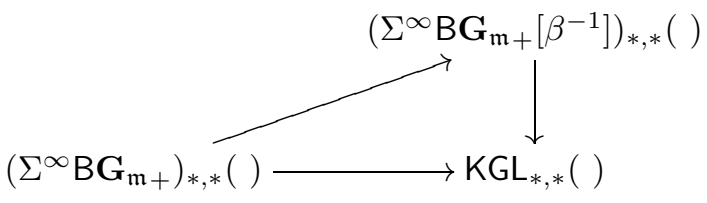

This diagram lifts uniquely to a commutative diagram of monoids in the motivic stable homotopy category, as asserted by the right hand side of (4.1), since for $K$-theory there exist no nontrivial phantom maps according to [6, Remark 9.8 (ii), (iv)]. 
On the left hand side of (4.1), recall that $\Sigma^{\infty} \mathrm{BG}_{\mathfrak{m}+} \rightarrow \mathrm{KGL}^{\beta}$ is a map of motivic symmetric ring spectra. For the discussion of $\mathrm{KGL}^{\beta} \rightarrow \Sigma^{\infty} \mathrm{BG}_{\mathfrak{m}+}\left[\beta^{-1}\right]$ we shall use the following model for the homotopy colimit. Let $\mathrm{E}$ be the stably fibrant replacement of $\Sigma^{\infty} \mathbf{B G}_{\mathfrak{m}}$ + obtained by first applying the functor $F$ levelwise and then applying the stabilization functor $Q$. Now define $\mathrm{E}\left[\beta^{-1}\right]$ as the diagonal spectrum of the naturally induced sequence

$$
\mathrm{E} \rightarrow \Sigma^{-2,-1} \mathrm{E} \rightarrow \cdots
$$

lifting the Bott tower (3.3). Here $\Sigma^{-2,-1} \mathrm{E}$ is realized as a shift, so that $\mathrm{E}\left[\beta^{-1}\right]_{n}=\mathrm{E}_{0}$ and its structure maps are given by multiplication with the Bott element. In level $n$ the map $\mathrm{KGL}^{\beta} \rightarrow \mathrm{E}\left[\beta^{-1}\right]$ is the canonical map

$$
\Omega^{4 n, 2 n} F\left(\mathrm{BG}_{\mathfrak{m}+} \wedge S^{4 n, 2 n}\right) \rightarrow \Omega^{2 \infty, \infty} F\left(\mathrm{BG}_{\mathfrak{m}+} \wedge S^{2 \infty, \infty}\right)=\mathrm{E}_{0}
$$

When $n=0$ the latter map corresponds via adjointness to the diagonal map in (4.1). The evident monoid structure on $\mathbf{B G}_{\mathfrak{m}+}$ induces a monoid structure on $\mathrm{E}_{0}$ and hence a naive multiplication on $\mathrm{E}\left[\beta^{-1}\right]$ given by

$$
\mathrm{E}\left[\beta^{-1}\right]_{m} \wedge \mathrm{E}\left[\beta^{-1}\right]_{n}=\mathrm{E}_{0} \wedge \mathrm{E}_{0} \rightarrow \mathrm{E}_{0}=\mathrm{E}\left[\beta^{-1}\right]_{m+n}
$$

Now from the construction of the ring structure on $\mathrm{KGL}^{\beta}$ it follows that $\mathrm{KGL}^{\beta} \rightarrow$ $\mathrm{E}\left[\beta^{-1}\right]$ respects the naive product.

\section{REFERENCES}

1. D.-C. Cisinski, F. Déglise. Triangulated categories of motives. arXiv:0912.2110.

2. B. I. Dundas, O. Röndigs, P. A. Østvær. Motivic functors. Doc. Math. 8:489-525 (electronic), 2003. MR2029171 (2004m:55011)

3. D. Gepner, V. Snaith. On the motivic spectra representing algebraic cobordism and algebraic K-theory. Doc. Math. 14:359-396 (electronic), 2009. MR2540697

4. J. F. Jardine. Motivic symmetric spectra. Doc. Math. 5:445-553 (electronic), 2000. MR:1787949 (2002b:55014)

5. F. Morel, V. Voevodsky. $\mathbf{A}^{1}$-homotopy theory of schemes. Inst. Hautes Études Sci. Publ. Math. 90:45-143 (2001), 1999. MR1813224 (2002f:14029)

6. N. Naumann, M. Spitzweck, P. A. Østvær. Motivic Landweber exactness. Doc. Math. 14:551593 (electronic), 2009.

7. N. Naumann, M. Spitzweck, P. A. Østvær. Chern classes, $K$-theory and Landweber exactness over nonregular base schemes, in Motives and Algebraic Cycles: A Celebration in Honour of Spencer J. Bloch, Fields Institute Communications, Vol. 56, 307-317, AMS, Providence, RI, 2009.

8. I. Panin, K. Pimenov, O. Röndigs. On Voevodsky's algebraic K-theory spectrum, in Algebraic Topology, Abel Symposium 2007, 279-330, Springer-Verlag, Berlin, 2009.

9. O. Röndigs, P. A. Østvær. Motives and modules over motivic cohomology, C. R. Math. Acad. Sci. 342:751-754, 2006. MR2227753(2007d:14043)

10. O. Röndigs, P. A. Østvær. Modules over motivic cohomology, Adv. Math. 219:689-727, 2008. MR 2435654(2009m:14026)

11. S. Schwede. An untitled book project about symmetric spectra. Available on the author's homepage, http://www.math.uni-bonn.de/ schwede.

12. M. Spitzweck, P. A. Østvær. The Bott inverted infinite projective space is homotopy algebraic K-theory, Bull. London Math. Soc. 41:281-292, 2009. MR2496504 
13. M. Spitzweck, P. A. Østvær. A Bott inverted model for equivariant unitary topological $K$ theory. To appear in Math. Scand.

14. V. Voevodsky. $\mathbf{A}^{1}$-homotopy theory. In Proceedings of the International Congress of Mathematicians, Vol. I (Berlin, 1998), Vol. I: 579-604 (electronic), 1998. MR1648048 (99j:14018)

Institut für Mathematik, Universität Osnabrück, 49069 Osnabrück, Germany

E-mail address: oroendig@math.uni-osnabrueck.de

Department of Mathematics, University of Oslo, P.O. Box 1053, Blindern, 0316 Oslo, NORWAY

E-mail address: markussp@math.uio.no

Department of Mathematics, University of Oslo, P.O. Box 1053, Blindern, 0316 Oslo, NORWAY

E-mail address: paularne@math.uio.no 